\title{
Towards internet of things modeling: a gateway approach
}

\author{
Ahmed B. Altamimi ${ }^{*}$ and Rabie A. Ramadan ${ }^{2}$
}

*Correspondence:

altamimi.a@uoh.edu.sa

${ }^{1}$ College of Computer

Science and Engineering,

University of Hail, Hail, Saudi

Arabia

Full list of author information

is available at the end of the

article

\begin{abstract}
Purpose: Internet of things (IoT) is an interaction between more than one network to facilitate communication. These networks by themselves are complex networks. Therefore, loT network is expected to grow at unprecedented scale involving other networks such as Mobile, VANET, and Wireless Sensor Networks (WSNs). In fact, modeling each network by itself is a complicated process. In addition, on a large scale, the communication among these networks increases the modeling complexity in which energy consumption could be critical due to large number of dropped messages. Therefore, this paper is a step forward towards modeling loT complex network through gateway deployment. The paper answers the question of how to deploy these gateways in a way that guarantees an efficient and adaptive communication.
\end{abstract}

Methods: Two models/methods are proposed and examined which are geographical based and mobile ferry based models. Due to the complexity of the deployment problem in reality, the deployment problem is treated as a complex adaptive problem and simulated through different sets of experiments and settings.

Results: The two methods have been compared through set of experiments using ONE simulator with the same number of employed gateways in the two methods. The experiments shows that ferry based model outperforms geographical based model with 29\% improvement in messages delivery probability. Additionally, when the number of mobile ferries are reduced by $34 \%$ compared to gateways that are distributed based on geographical area, the mobile ferries approach still outperform geographical area based approach when it comes to messages delivery probability.

Conclusions: The paper presents the two methods to model the complex internet of things environment and its sub networks interaction. The paper concludes that employing mobile ferries as gateways is better than deploying gateways based on geographical area when the sub networks interaction is facilitated in loT network.

Keywords: Gateways deployment, Geographical based modeling, Ferries based modeling

\section{Background}

IoT is defined as a network that allows sub networks including sensor, mobile, and vehicular networks to communicate with each other. Wireless sensor network is defined as a group of distributed sensors that collect data regarding physical or environmental conditions, such as temperature, sound, pressure etc. and to cooperatively pass these data through the network to a main location. Figure 1 shows the reporting style of wireless 

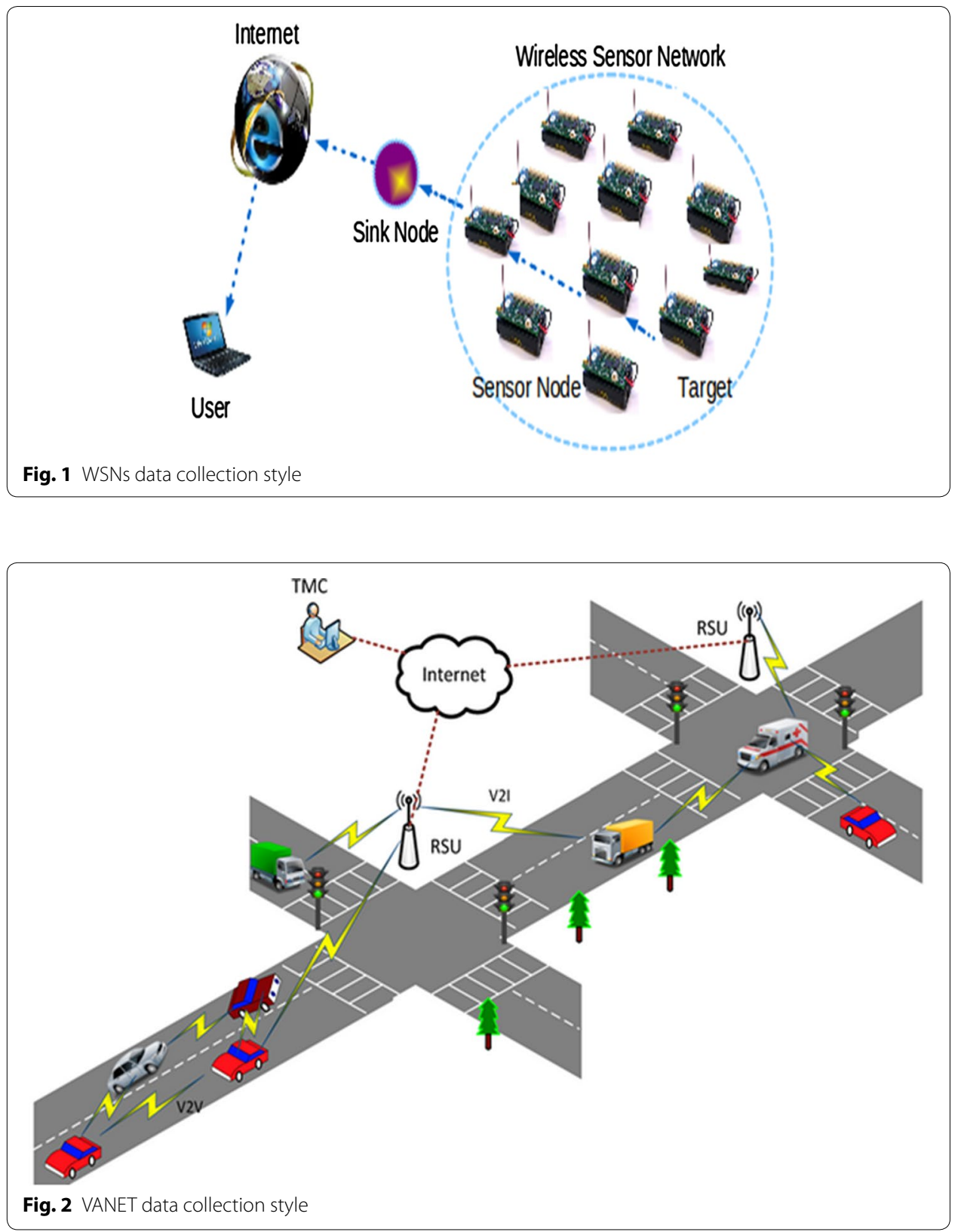

sensor network (WSNs), where sink nodes are used for data collections. WSNs applications include monitoring forest fire and controlling home temperature. Vehicular network on the other hand is a way of facilitating communications between vehicles. Messages are exchanged between vehicles and also between vehicles and road side units as a data collection center in such network. Figure 2 shows the use of road side units for data collections in VANET. Such network is beneficial in warning drivers of any coming traffic and delivering messages to areas where infrastructure networks can't reach them. The last type of network that would be part of IoT environment in this paper is a wireless mobile network where messages are exchanged through mobiles and mobiles could act 
like a message hop. One application of this network is in the military field where a network with fixed infrastructure is difficult to be installed and maintained.

The integration between different networks in IoT can bring the benefits and the characteristics of each network to the IoT environment. For example, sensor networks are mainly used for sensing and collecting data. Additionally, it is usually considered as a static network. Whereas, mobile or vehicular networks are often employed for unicast communication between nodes or vehicles. Additionally, it is known for its mobiles behaviors. Bringing these different characteristics and benefits in one environment is attractive. An international recognition of IoT increases its attractiveness too. The US national Intelligence Council lists the IoT among the six technologies that may impact US national power by 2025; in addition, the European Commission is financing several research projects on the subject within the Framework Programme; moreover, in Asia increasing research efforts are being devoted to the definition of technologies for the IoT (Iera et al. 2010).

IoT is a complex system in which its complexity comes from number of networks involved, the interconnectivity of the components of the IoT, and the interaction with the environment. The nonlinear spatio-temporal interactions among IoT component makes it an complex adaptive systems (CAS), complex adaptive communication networks and environments (CACOONS) (Niazi and Hussain 2009, 2013; Fatima and Ali Shah 2015; Niazi 2009; Niazim and Hussain 2013; Laghari and Niazi 2016). Such kind of systems are modeled usually through simulation due to their complexity.

Consequently, the main concern of this work to be solved is to model the interaction between different networks in IoT environment, namely, mobile, vehicular, and wireless sensor networks. Mobile and vehicular network is defined as the groups of nodes or vehicles that use their cooperation to facilitate communication without the use of infrastructure. The main proposed method to integrate mobile/vehicular networks to IoT is the gateway, such as, access points to allow different networks to communicate (Bessis et al. 2013) and (Bellavista et al. 2013). WSNs, on the other hand, normally are a centralized network, where a node or group of nodes are in charge of collecting the sensed data. Gateway could be possible solution to connect WSN to the IoT environment as presented in Bessis et al. (2013) and Bellavista et al. (2013).

The paper is organized as follows: the next section states some of the related work; "IoT gateway modeling" section explains the modeling of IoT proposed in this paper; "Simulation results" section includes the simulation results; the paper concludes in "Conclusion" section.

\section{Related work}

Internet of things (IoT) has many applications that help to solve issues related to city and health services. The services include monitoring street parking, heart problem, etc. as an integrated solution. This allows IoT to be the subject for many research articles (Wei et al. 2013; Al Ayubi 2209; Gao and Liu 2011; Shi and Rekeczky 2007; Freitas et al. 2009; Hong et al. 2010; Zorzi et al. 2010; Yue et al. 2014; Botta et al. 2016; Khodkari and Maghrebi 2016; Tsai et al. 2014; Li et al. 2016; Raza et al. 2013; Sheng et al. 2013) analyzing IoT from different prospective. For example, the authors of (Yue et al. 2014; Botta et al. 2016; Khodkari and Maghrebi 2016) propose the use of cloud storage in IoT. 
Additionally, some security aspects have been addressed in ( $\mathrm{Li}$ et al. 2016; Raza et al. 2013). However, incorporating any concept including cloud storage and security concern will require modeling of IoT environment.

IoT modeling has been addressed in many previous works (Bessis et al. 2013; Bellavista et al. 2013; Huang and Li 2010; Riedel et al. 2010; Seel et al. 2010). However, most of the existing modeling is concerned about connecting the RFID technology to the IoT environment (Huang and Li 2010; Riedel et al. 2010; Seel et al. 2010). Bessis et al. (2013) and Bellavista et al. (2013) study the integration of mobile, vehicular and sensor networks in an IoT environment to some extent. In both articles, a gateway including access point is proposed as a mean to integrate these networks together. However, their proposal was only theoretically examining the integration of two networks. On the other hand, the research in this paper will discuss and simulate the applicability and the integration of the three networks together which are mobile, vehicular and sensor networks.

Recently, gateway deployment/placement in wireless networks has been an active research field. Major work in this area include how to deploy gateways in wireless sensor networks (Ibrahim et al. 2007; Beutel et al. 2009; Youssef and Younis 2007) or/and wireless mesh networks (Lakshmanan et al. 2006; Fan et al. 2007; Benyamina et al. 2009; Bing et al. 2007; Aoun et al. 2006). The gateway in both cases was deployed only for static networks. Whereas, IoT may include static and dynamic nodes. This makes the gateways deployment even harder in IoT environment in comparison to static networks.

In literature, gateway deployment is mainly proposed through a gateway per each network cluster or optimizes a performance metric including throughput or delay to find out the best possible gateway deployment strategy (Ibrahim et al. 2007; Beutel et al. 2009; Youssef and Younis 2007; Lakshmanan et al. 2006; Fan et al. 2007; Benyamina et al. 2009; Bing et al. 2007; Aoun et al. 2006). It has been noticed that both methods could work just fine for static networks. However, based on our experience in IoT scenario, it might be a good idea to take the advantage of previous proposed protocols in delay tolerant networks, where a message ferry can be employed for routing (Tariq et al. 2006; Miura et al. 2010; Xue et al. 2012) in IoT environment. Other work (Leontiadis and Mascolo 2007; Sidera and Toumpis 2013) propose that routing is based on petrographical area. Gateway deployment as message ferry or based on geographical area both can be employed to resolve the gateway deployment problem in IoT environment.

\section{IoT gateway modeling}

IoT modeling is an important problem to be analyzed. Its importance can be seen from the number of IoT applications that can be implemented with a successful modeling of the environment. Two IoT modeling methods are discussed and simulated in this paper. Gateway deployment based on geographical area and based on mobile ferries are presented.

In the geographical method, gateways are geographically distributed to fully ensure the connectivity among the three networks. This could be implemented by ensuring that each two circles intersect in one point at least, where a circle represent the gateway communication range. Figure 3 shows twelve sub areas, as an example, to fully cover the working area. These sub areas can be utilized to install the gateways where full connectivity must be guaranteed. This method ensures the full connectivity between their 


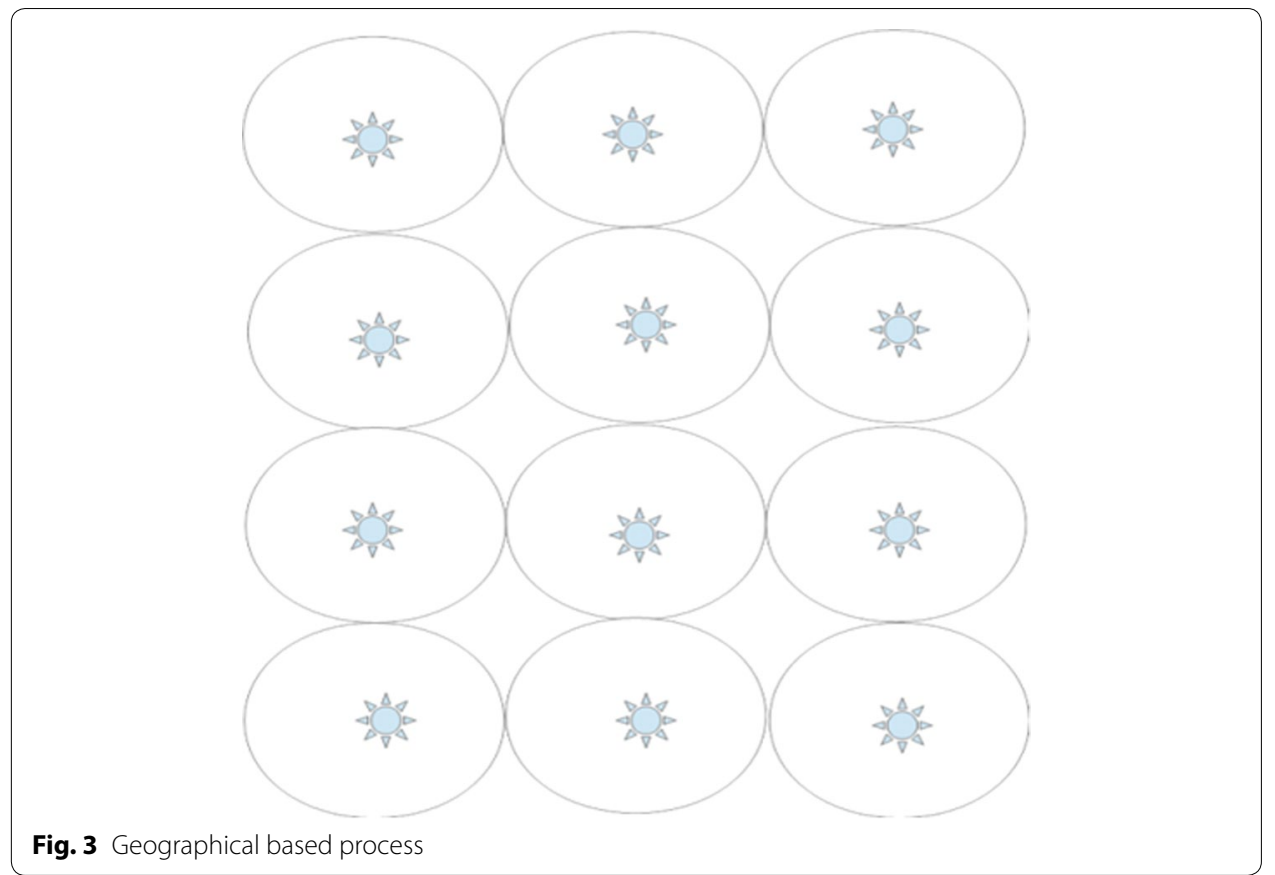

gateways based on the solution of Circle and Sphere Packing problems stated in Melissen and Schuur (2000), Hifi and M'Hallah (2009), Litvinchev and Ozuna (2014), Ramadan and Abdel-Mageid (2010), Birgin et al. (2005).

The problem of circle packing tries to minimize the waste of divided rectangle or square plan to circles. The gateways deployment in this paper is inspired from the work done in Birgin et al. (2005) to pack the area under study, as it is shown in the evaluation section. The authors solved the packing problem by dividing the rectangular box $[0$, $\left.d_{1}\right] \times\left[0, d_{2}\right]$ to $k$ circles of radius $r$ in such a way that the intersection between any pair of circles $i$ and $j, i \neq j$, is at most one point, i.e., the circles are not overlapped. Therefore, given $k, r, d_{1}$ and $d_{2}$, the goal is to determine $\mathrm{p}^{1}, \ldots, \mathrm{p}^{\mathrm{k}} \in\left[\mathrm{r}, \mathrm{d}_{1}-\mathrm{r}\right] \times\left[\mathrm{r}, \mathrm{d}_{2}-\mathrm{r}\right]$ solving the problem:

$$
\begin{aligned}
& \text { Minimize } \sum \max \left(0,(2 r)^{2}\left\|p^{i} p^{j}\right\|_{2}^{2}\right)^{2} \\
& \text { subject to } \\
& \mathrm{r} \leq \mathrm{p}_{1}^{\mathrm{i}} \leq \mathrm{d}_{1}-\mathrm{r} \text {, and } \\
& \mathrm{r} \leq \mathrm{p}_{2}^{\mathrm{j}} \leq \mathrm{d}_{2}-\mathrm{r}, \quad \text { for } \mathrm{i}=1, \ldots, \mathrm{k} \text {. }
\end{aligned}
$$

Points $\mathrm{p}^{1}, \ldots, \mathrm{p}^{\mathrm{k}}$ are the centers of the desired circles. One circle will be packed at the beginning, and the packing will continue to accommodate more circles, until no more space is available inside the rectangular.

Geographical based method has a fixed infrastructure nature where gateways are placed to cover one area. This method centrally controlled and synchronized. This allows for non-overlapping transmission between gateways and mobile nodes. Additionally, the load of messages transmission would be balanced since each gateway is taking care of one circle. Moreover, centralized controller allows easy deployment of security and quality of services measures on the employed gateways. However, it raises the challenge of 


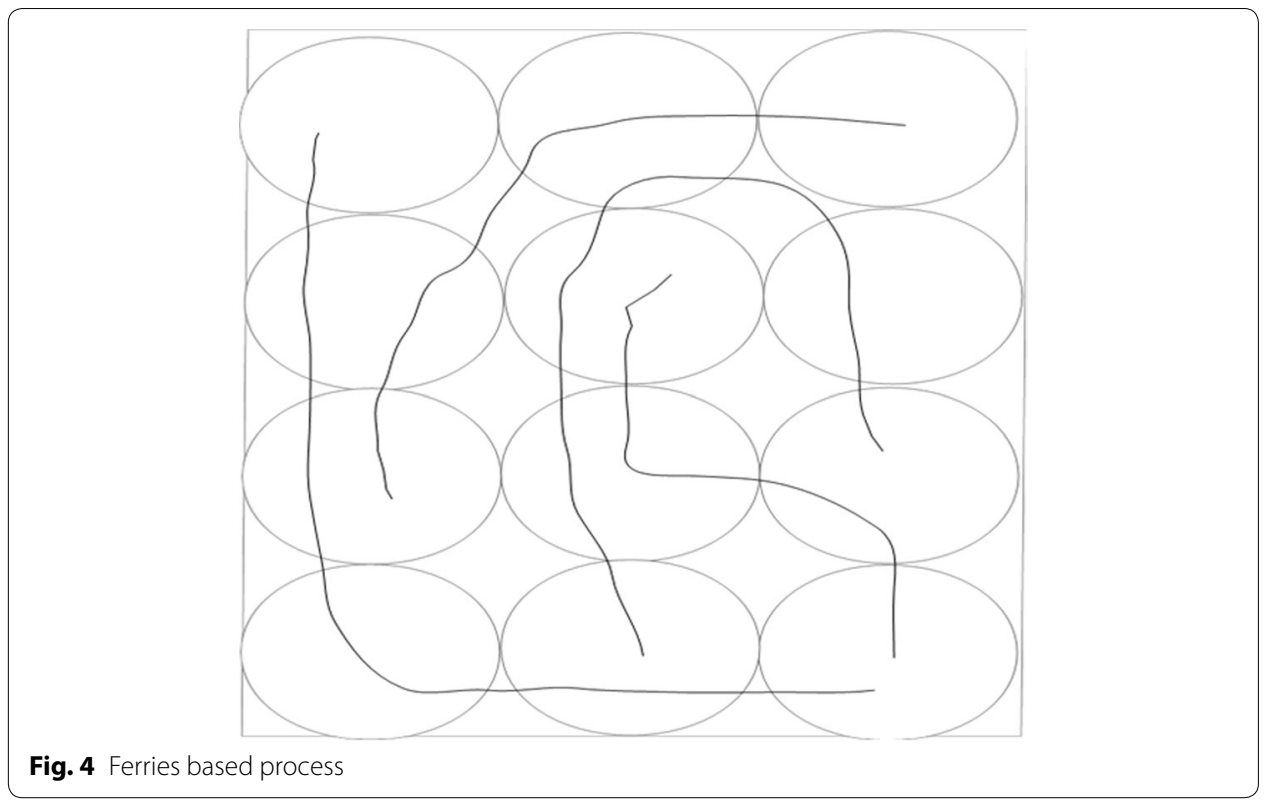

one point of failure, where if one of the gateways is down, it would risk the whole network of being down.

The other method where gateways are based on mobile ferries; the mobility of the ferries guarantees the reachability of the ferries to sub networks including mobile, vehicular and sensor network in the IoT environment. This helps fast and efficient messages transfer between sub networks in the system. Figure 4 shows the concepts of ferries employment in IoT environment. With the same 12 sub divided areas that were employed in the geographical based method with fixed gateways, a ferry can travel from one sub area to another. Generally, ferries can travel in any existing paths. Four routes are shown in the figure as an example of how a ferry travels inside the network area. Gateways in the four paths example can help to forward messages from one sub-network to another.

Mobile ferries method has an infrastructure less nature where gateways are freely moved on the network area. This method guarantees flexibility of its gateways movements. Additionally, it is a reliable method for message delivery since the failure of one gateway, won't lead to failure of the network. However, gateways in this method would require more resources to be consumed including energy consumption.

The above two methods are compared and analyzed by simulation in the next section. The comparison includes their delivery probability of created messages. Message delivery probability is defined as the number of delivered messages to the destinations to the number of created messages by source nodes. This reflects the better choice of the two methods to be implemented in the IoT environment.

\section{Simulation results}

This section contains the details of our Complex Adaptive COmmunicatiOn Networks and environmentS (CACOONS) simulation to the two previously proposed for IoT modeling. The section starts by explaining the details of the used co-simulator and the simulation settings followed by examining the performance of the proposed methods. 


\section{Simulation environment}

It is clear that simulating three different networks with different topologies and settings as well as requirement is a very hard task. In addition, selecting a suitable solution is also a challenging task. In this paper, ONE simulator (Keränen et al. 2009) has been chosen and adapted to fit the requirements of the three different networks, WSNs, VANET, and mobile networks. ONE is a discrete event simulation package. It combines movement modeling, routing, visualization and reporting. Mobility models determine node movement within the simulation environment. The random waypoint model (RWPM) is widely used and is based on random directions and speeds. However, this random node movement is unrealistic when mobile devices are carried by humans. It is more pragmatic to assume that nodes move towards a specific destination, then another destination, and so on. These destinations are typically particular locations such as malls, restaurants or schools, and so are called points of interest (PoI). The more realistic shortest path movement model (SPMM) (Keränen et al. 2010) has nodes moving towards particular locations, and so is employed here. In SPMM (Keränen et al. 2010), nodes travel through the shortest path between two points where PoIs are located in the map, such as restaurants or shopping mall, as destinations for moving nodes.

The simulation parameters employed here are carried out based on the realistic environment described in Altamimi and Gulliver (2012) where Helsinki City Scenario (HCS) (Keränen et al. 2010) map is used. The scenario has nodes moving in a part of the downtown Helsinki area including its roads, pedestrian walkways and connectivity traces from the real time location data of Helsinki trams. With HCS, node mobility is based on simulating 50 mobile users moving by foot, 30 by car, and 6 by trams in the streets of downtown Helsinki. Additionally, three sensor networks are randomly deployed in three locations; each network consists of 10 nodes. Each node represents a user moving with realistic speed along the shortest paths between different POIs and random locations. The trams follow real tram routes in Helsinki. The simulation area is designed to be $4500 \times 3400 \mathrm{~m}^{2}$ size. The simulation environment parameters are summarized in Table 1.

The gateways however, are differently deployed based on the used method. 12 gateways are geographically placed to cover the simulation area based on the proposed method in Birgin et al. (2005) when geographical based deployment method is employed. The same number of mobile ferries are deployed when gateways deployment are based on mobile ferries.

\section{Performance results}

In this section, simulation experiments are conducted to examine the performance of the two proposed methods. It has been assumed that messages are routed toward gateways

Table 1 Simulation environment parameters

\begin{tabular}{ll}
\hline Parameter & Value \\
\hline Transmit rate & $250 \mathrm{kbps}$ \\
Transmit range & $50 \mathrm{~m}$ \\
Message size & $50-150 \mathrm{~kb}$ \\
\hline
\end{tabular}


when they want to be exchanged with different sub network. For instance, when a node in sensor network wants to exchange message to a node in vehicular network, it has to be directed to a gateway. The simulation has been run twice. One is to compare the network performance when geographical based gateway deployment is employed with high speed ferries gateways deployment. The other run is to examine the delivery probability of the network when geographical based gateway deployment is employed to when low speed ferries are used.

Figure 5 shows that the network with high speed ferries have a better delivery probability with $29 \%$ improvement to when gateways are geographically deployed. This is because high speed ferries are more likely to meet the three sub networks in multipaths, where geographical based gateways deployments, it is likely to have only one path to reach the final destination.

Figure 6 shows that the network with low speed ferries have a similar delivery probability to when gateways are geographically deployed. This means that the speed of mobile ferries would determine whether to deploy the gateways in IoT environment based on geographical based or based on random deployment of mobile ferries.

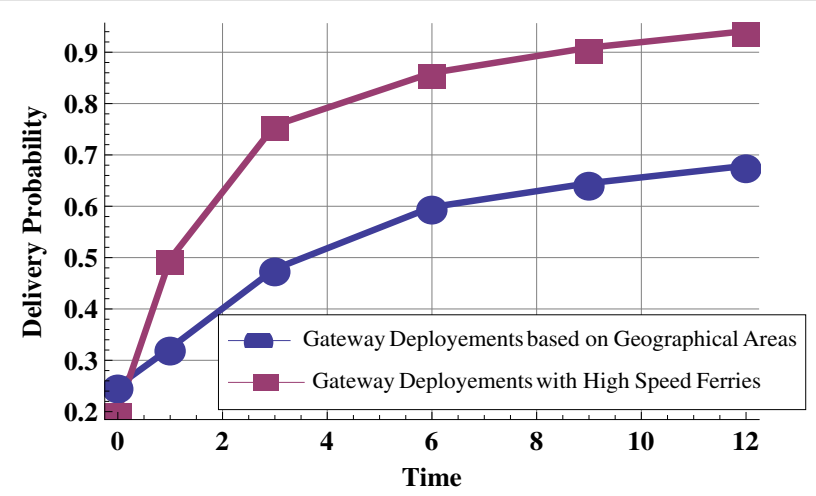

Fig. 5 Delivery probability comparison when gateways deployment is with high speed ferries to geographical based deployment

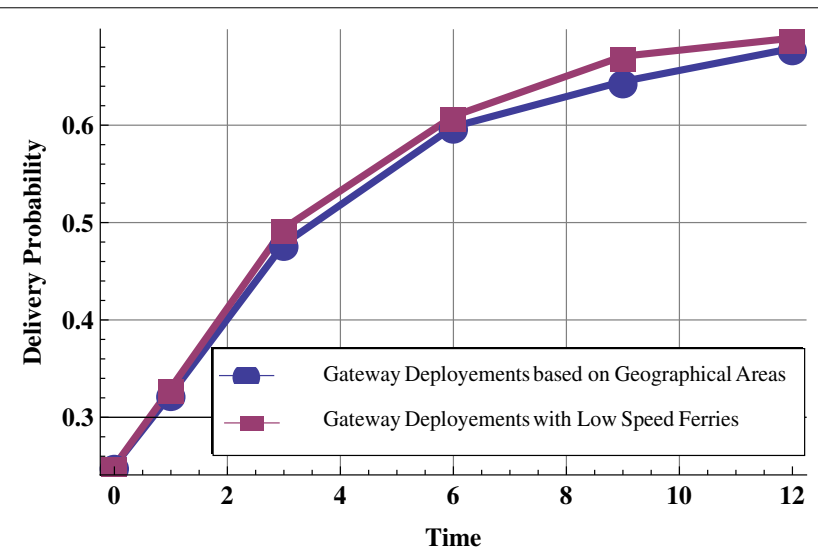

Fig. 6 Delivery probability comparison when gateways deployment is with low speed ferries to geographical based deployment 
Figures 5 and 6 show that gateways deployment as mobile ferries in IoT environment leads to a better delivery probability network than gateways deployed based on geographical area in the same environment. The improvement of delivery probability in such environment when mobile ferries are employed is due to the ability of gateways to encounter the three sub networks, namely vehicular, sensor and mobile networks nodes. Whereas, geographical area deployment based ensures that gateways belongs to one region when one sub network might be in there. Therefore, the interactions between the three sub networks and messages delivery would be easier to achieve in case of mobile ferries gateways, compared to geographical area deployment based.

The previous discussion shows the improvement of delivery performance when ferries are employed compared to geographical area based method with the number of gateways is equal. The question that is raised here, when the two methods will have similar performance under different number of installed gateways. The simulation have been run for multiple number of gateways in the ferries based method to reach a comparable performance when geographical based method is employed.

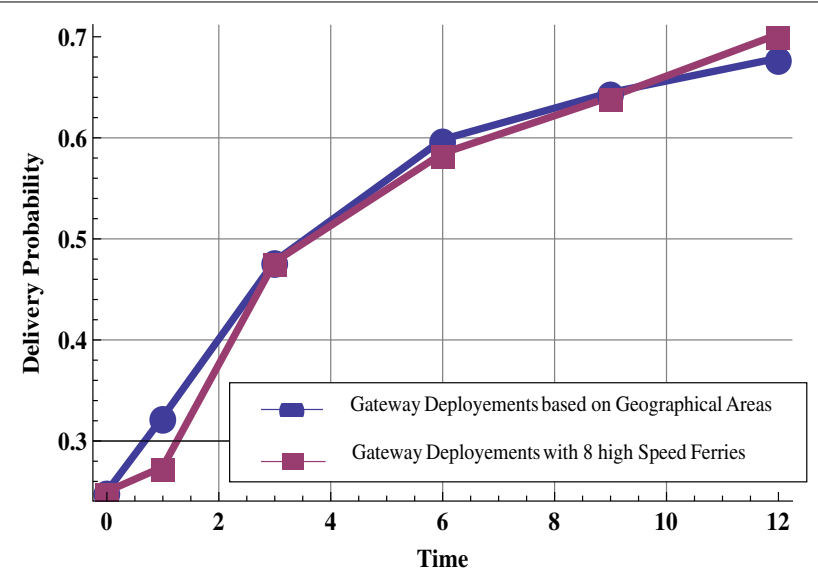

Fig. 7 Delivery probability comparison when gateways deployment is with 8 high speed ferries to 12 geographical based deployment

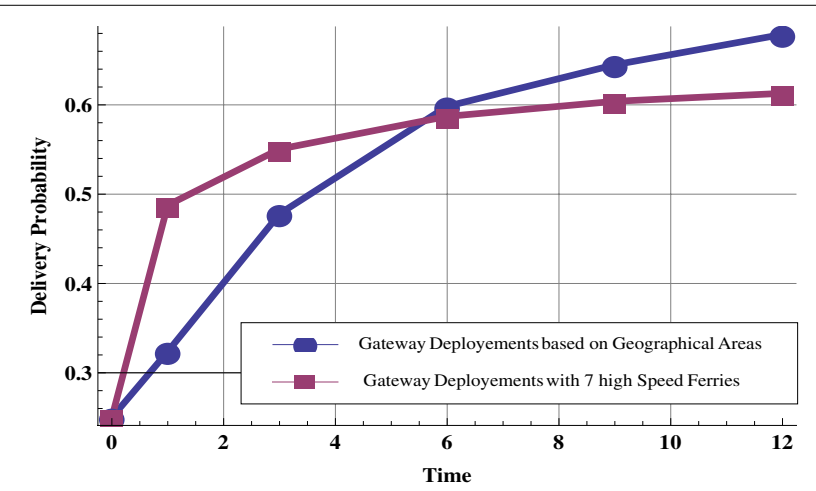

Fig. 8 Delivery probability comparison when gateways deployment is with 7 high speed ferries to 12 geographical based deployment 
Figure 7 shows that ferries method still outperforms geographical based method when the number of gateways are decreased from 12 to 8 (34\%). Whereas the geographical based method outperforms the ferries based method when the number of gateways in ferries based methods are decreased by $40 \%$ or to 7 gateways as shown in Fig. 8 . This concludes that the ferries based method still outperforms the geographical based method when the number of gateways in ferries method is less than geographical based method by $34 \%$.

\section{Conclusion}

This paper presents two methods to model the sub networks interaction in the complex IoT environment. Gateways based on mobile ferries and geographical based are compared and empirically analyzed. The simulation shows that mobile ferries gateways deployment improves the IoT environment delivery probability by $29 \%$ when a proper mobile ferries speed have been chosen. Additionally, with fewer gateways by $34 \%$, the mobile ferries outperforms the geographical based method in terms of delivery probability.

\section{Authors' contributions}

AA and RR conceived, designed and developed the idea behind the two models comparison. AA performed the simulations and analyzed the simulation results. AA and RR wrote the paper. Both authors read and approved the final manuscript.

\section{Author details}

${ }^{1}$ College of Computer Science and Engineering, University of Hail, Hail, Saudi Arabia. ${ }^{2}$ College of Computer Science and Engineering, University of Hail and Cairo University, Hail 81451, Hail, Saudi Arabia.

\section{Competing interests}

Both authors declare that they have no competing interests.

Received: 19 September 2016 Accepted: 25 October 2016

Published online: 02 November 2016

\section{References}

Al Ayubi S (2209) A framework of spreading disease monitoring system model. In: International conference on instrumentation, communications, information technology, and biomedical engineering (ICICl-BME). pp 1-5, 23-25 Altamimi A, Gulliver T (2012) On routing protocols using mobile social networks. Int J Wirel Mobile Comput 7(3):1-11 Aoun B, Boutaba R, Iraqi Y, Kenward G (2006) Gateway placement optimization in wireless mesh networks with QoS constraints. IEEE J Sel Areas Commun 24(11):2127-2136

Bellavista P, Cardone G, Corradi A, Foschini L (2013) Convergence of MANET and WSN in loT urban scenarios. IEEE Sens J 13(10):3558-3567

Benyamina D, Hafid A, Gendreau M (2009) Optimal placement of gateways in multi-hop wireless mesh networks: a clustering-based approach. In: IEEE 34th conference on local computer networks. pp 625-632

Bessis N, Xhafa F, Varvarigou D, Hill R, Li M (eds) (2013) Internet of things and inter-cooperative computational technologies for collective intelligence. Springer, Berlin. ISBN:978-3-642-34952-2

Beutel J, Rmer K, Ringwald M, Woehrle M (2009) Deployment techniques for sensor networks. In: Sensor networks. Springer, Berlin, pp 219-248

Bing H, Bin X, Agrawal D (2007) Optimizing the internet gateway deployment in a wireless mesh network. In: IEEE international conference on mobile adhoc and sensor systems. pp 1-7

Birgin E, Martnez J, Ronconi D (2005) Optimizing the packing of cylinders into a rectangular container: a nonlinear approach. Eur J Oper Res 160(1):19-33

Botta A, de Donato W, Persico V, Pescapé A (2016) Integration of cloud computing and internet of things: a survey. Future Gener Comput Syst 56(1):684-700

Fan L, Yu W, Xiang-Yang L (2007) Gateway placement for throughput optimization in wireless mesh networks. In: IEEE international conference on communication. pp 4955-4960

Fatima B, Ali Shah M (2015) Self organization based energy management techniques in mobile complex networks: a review. Complex Adapt Syst Model 3:2-10

Freitas E, Allgayer R, Wehrmeister M, Pereira C, Larsson T (2009) Supporting platform for heterogeneous sensor network operation based on unmanned vehicles systems and wireless sensor nodes. In: IEEE intelligent vehicles symposium. pp 786-791 
Gao C, Liu J (2011) Modeling and predicting the dynamics of mobile virus spread affected by human behavior. In: IEEE international symposium on a world of wireless, mobile and multimedia networks (WoWMoM). pp 1-9, 20-24

Hifi M, M'Hallah R (2009) A literature review on circle and sphere packing problems: models and methodologies. Adv Oper Res 3:1-22

Hong S, Kim D, Ha M, Bae S, Park S, Jung W, Kim J (2010) SNAlL: an IP-based wireless sensor network approach to the internet of things. IEEE Wirel Commun 17(6):34-42

Huang Y, Li G (2010) Descriptive models for Internet of Things. In: International conference on intelligent control and information processing (ICICIP). p 483-486

Ibrahim S, Jun-Hong C, Ammar R (2007) Surface-level gateway deployment for underwater sensor networks. In: Military communications conference. p 1-7

lera A, Floerkemeier C, Mitsugi J, Morabito G (2010) The internet of things. IEEE Wirel Commun 17(6):8-9

Keränen A, Ott J, Kärkkäinen T (2009) The ONE simulator for DTN protocol evaluation. In: proceedings of the 2nd international conference on simulation tools and techniques, Rome, Italy, p 1-10

Keränen A, Kärkkäinen T, Ott J (2010) Simulating mobility and DTNs with the ONE. J Commun 10(2):92-105

Khodkari H, Maghrebi S (2016) Necessity of the integration Internet of Things and cloud services with quality of service assurance approach. Bulletin de la Société Royale des Sciences de Liège 85(1):434-445

Laghari S, Niazi A (2016) Modeling the internet of things, self-organizing and other complex adaptive communication networks: a cognitive agent-based computing approach. PLOS ONE 11:1-10

Lakshmanan S, Sundaresan K, Sivakumar R (2006) On multi-gateway association in wireless mesh networks. In: IEEE workshop on wireless mesh networks. pp 64-73

Leontiadis I, Mascolo C (2007) GeOpps: geographical opportunistic routing for vehicular networks. In: IEEE international symposium on a world of wireless, mobile and multimedia networks. pp 1-6

Li S, Tryfonas T, Li H (2016) The internet of things: a security point of view. Internet Res 26(2):337-359

Litvinchev I, Ozuna E (2014) Approximate packing circles in a rectangular container: valid inequalities and nesting. J Appl Res Technol 12(4):1-10

Melissen J, Schuur P (2000) Covering a rectangle with six and seven circles. In: In proceedings of the 5 th twente workshop on graphs and combinatorial optimization. Elsevier Science Publishers, Amsterdam, pp 149-156

Miura H, Nishi D, Matsuda N, Taki H (2010) Message ferry route design based on clustering for sparse ad hoc networks. In: Knowledge-based and intelligent information and engineering systems, Springer, Berlin, pp 637-644

Niazi M (2009) Complex adaptive systems modeling: a multidisciplinary roadmap. Complex Adapt Syst Model 1(1):1-14

Niazi M, Hussain A (2009) Agent-based tools for modeling and simulation of self-organization in peer-to-peer, ad hoc, and other complex networks. In: IEEE Communications Magazine, vol 47, pp 166-73

Niazi M, Hussain A (2013) Complex adaptive communication networks and environments: part 2. Simulation 89(7):787-789

Niazim M, Hussain A (2013) Complex adaptive communication networks and environments: part 1. Simulation 89(5):559-561

Ramadan R, Abdel-Mageid S (2010) Efficient deployment of connected sensing devices using circle packing algorithms. In: 2010 International conference on autonomous and intelligent systems (AIS). pp 1-6

Raza S, Shafagh H, Hewage K, Hummen R, Voigt T (2013) Lithe: lightweight secure CoAP for the internet of things. IEEE Sens J 13(10):3711-3720

Riedel T, Yordanov D, Fantana N, Scholz M, Decker C (2010) A model driven internet of things. In: Seventh international conference on networked sensing systems (INSS). pp 265-268

Seel C, Schimmelpfennig J, Mayer D, Walter P (2010) Conceptual modeling of complex events of the internet of things. In: eChallenges. pp $1-8$

Sheng X, Tang J, Xiao X, Xue G (2013) Sensing as a service: challenges, solutions and future directions. IEEE Sens J 13(10):3733-3741

Shi B, Rekeczky C (2007) Sensor integration in autonomous systems. In: IEEE international symposium on circuits and systems. p 601-604, 27-30

Sidera A, Toumpis S (2013) Delay tolerant firework routing: a geographic routing protocol for wireless delay tolerant networks. EURASIP J Wireless Com Network 23. doi:10.1186/1687-1499-2013-23

Tariq M, Ammar M, Zegura E (2006) Message ferry route design for sparse ad hoc networks with mobile nodes. In: Proceedings of the 7th ACM international symposium on mobile ad hoc networking and computing. p 37-48

Tsai C, Lai C, Chiang M, Yang L (2014) Data mining for internet of things: a survey. IEEE Commun Surv Tutor 16(1):77-97

Wei X, Valler NC, Faloutsos M, Neamtiu I, Prakash BA, Faloutsos C (2013) Smartphone viruses propagation on heterogeneous composite networks. In: IEEE 2nd network science workshop (NSW). p 106-109

Xue L, Liu J, Peng J (2012) An adaptive message ferry routing algorithm for delay tolerant networks. In: IEEE 14th international conference on communication technology. p 699-703

YoussefW, Younis M (2007) Intelligent gateways placement for reduced data latency in wireless sensor networks. In: IEEE international conference on communication. p 3805-3810

Yue H, Guo L, Li R, Asaeda H, Fang Y (2014) DataClouds: enabling community-based data-centric services over the internet of things. IEEE Internet Things J 1 (5):472-482

Zorzi M, Gluhak A, Lange S, Bassi A (2010) From today's INTRAnet of things to a future INTERnet of things: a wireless- and mobility-related view. IEEE Wirel Commun 17(6):44-51 